\title{
APORTACIONES DE LA TERAPIA DE ACEPTACIÓN Y COMPROMISO AL TRABAJO CON MADRES Y PADRES DE NIÑAS Y NIÑOS ATENDIDOS EN SALUD MENTAL: EXPERIENCIAS DE LA INTERVENCIÓN GRUPAL
}

\section{CONTRIBUTIONS OF ACCEPTANCE AND COMMITMENT THERAPY (ACT) IN WORK WITH THE PARENTS OF CHILDREN IN MENTAL HEALTH SERVICES: EXPERIENCES FROM GROUP INTERVENTIONS.}

\author{
Mónica Díaz de Neira Hernando, Carmen Vidal Mariño, \\ Susana González Rueda y Pedro Gutiérrez Recacha \\ Fundación Jiménez Díaz, CEP Argüelles, Madrid, España
}

Cómo referenciar este artículo/How to reference this article:

Díaz de Neira Hernando, M., Vidal Mariño, C., González Rueda, S. y Gutiérrez Recacha, P. (2016). Aportaciones de la Terapia de Aceptación y Compromiso al Trabajo con Madres y Padres de Niñas y Niños Atendidos en Salud Mental: Experiencias de la Intervención Grupal. Revista de Psicoterapia, 27(104), 149-165.

\begin{abstract}
Resumen
En la literatura cientifica se pueden encontrar distintas propuestas de grupos destinados al entrenamiento de madres y padres de niños y niñas con problemas comportamentales, predominando aquéllas que se basan en la Terapia de Conducta. El desarrollo de las Terapias Contextuales ha supuesto un cambio poniendo la meta del tratamiento no en la reducción/eliminación de sintomas, sino en que la persona logre una vida con más significado, usando para ello estrategias de aceptación. En particular, la Terapia de Aceptación y Compromiso (ACT) ha aportado una teoría sobre la cognición y el lenguaje, destacando su influencia en la regulación del comportamiento, integrando los valores en el tratamiento y desarrollando estrategias terapéuticas para enfrentarse de forma diferente a las emociones y pensamientos aversivos que funcionan como barreras para el cambio. El presente artículo describe la propuesta de un programa de diez sesiones basado en la ACT así como la valoración que hicieron los participantes de la utilidad del programa al aplicarlo en un Centro de Salud entre los años 2012 y 2015, a un total de 69 familias.
\end{abstract}

Palabras clave: ACT, Grupos de Padres, Terapia de Conducta, Problemas de Conducta

\footnotetext{
Fecha de recepción: 04/04/2016. Fecha de aceptación v1: 05/05/2016. Fecha de aceptación v2: 13/06/2016 Correspondencia sobre este artículo: E-mail: monicadiazdeneira@gmail.com

Dirección postal: Mónica Díaz de Neira Hernando. Fundación Jiménez Díaz, CEP Argüelles, C/ Quintana, 11, 28008 Madrid, España.

C 2016 Revista de Psicoterapia
}

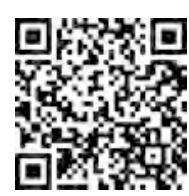




\section{Abstract}

Scientific literature proposes different training programs for parents of children with behavioral problems, predominantly those based on Behavior Therapy. The development of contextual therapies has meant a turning point, changing the treatment goal from symptom reduction/elimination to the achievement of a more meaningful life by means of acceptance strategies. Specifically, Acceptance and Commitment Therapy (ACT) has provided a theory of cognition and language, highlighting their influence on behavioral regulation, integrating values in the treatment, and developing therapeutic strategies to deal differently with those emotions and aversive thoughts that may be acting as barriers to change. The current work describes the proposal for a 10-session ACT-based training program and reports the results obtained when applied to 69 families in a health center between 2012 and 2015.

KeyWords: Acceptance and Commitment Therapy, ACT, Group-Based Parent Programs, Behaviour Therapy, Behavioural Disorders 


\section{Introducción}

Hasta la fecha, se han propuesto programas de entrenamiento de madres y padres que parten de diversas orientaciones psicoterapéuticas, destacando los basados en la Terapia de Conducta. El presente artículo se centra en la puesta en práctica de un grupo para madres y padres tomando como base teórica la Terapia de Aceptación y Compromiso (ACT). Se analizan los resultados obtenidos después de llevar a cabo nueve ediciones del mismo en un Centro de Salud entre los años 2012 y 2015. En el presente artículo se revisarán las distintas perspectivas sobre grupos de padres y madres que se pueden encontrar en la literatura científica; se abordarán, desde una perspectiva histórica, las terapias contextuales, y en particular la Terapia de Aceptación y Compromiso; se analizarán las intervenciones en población infantil desde esta orientación; se describirán los objetivos y las características de la intervención; se presentará la valoración y adherencia al programa de los participantes y finalmente se discutirán las implicaciones y fortalezas de este enfoque.

\section{Los grupos de entrenamiento para madres y padres: perspectivas, propuestas y resultados.}

En las últimas décadas se han desarrollado múltiples programas de entrenamiento de madres y padres, que podrían catalogarse dentro de dos grandes orientaciones teóricas diferentes: la aproximación "democrática" o "basada en las relaciones interpersonales" y la aproximación "conductual" (Barlow, Coren y Stewart-Brown, 2002; Richardson y Joughin, 2002). La primera se apoya en teorías humanistas y adlerianas, y basa su intervención principalmente en la comunicación familiar y en los procesos de interacción ocurridos entre los miembros de la familia. En cambio, la aproximación conductual, utiliza técnicas derivadas de las teorías del aprendizaje para que se produzcan cambios comportamentales positivos, realizando las manipulaciones ambientales necesarias (Sanders y Dadds, 1993; Silvares, 1995).

Robles y Romero (2011) describen la revisión de Todres y Bunston (1993), en la que incluyeron 62 trabajos, encontrando en el grupo de programas conductuales mejores resultados que en el de humanistas y el de adlerianos.

Según Marinho y Ferreira (2000), el programa grupal de entrenamiento a padres más ampliamente evaluado ha sido el propuesto por Webster-Stratton (Webster-Stratton, 1994; Webster-Stratton, 1996; Webster-Stratton y Hammond, 1997) que utiliza métodos de aprendizaje por observación del modelo a través de una serie de programas grabados sobre habilidades de los padres. Se trata de un tratamiento "bien establecido" de los 82 estudios revisados sobre tratamiento a niños con problemas conductuales (Brestan y Eyberg, 1998).

En el trabajo de Furlong et al (2012), tras revisar sistemáticamente 13 ensayos con un total de 1078 participantes de grupos de crianza grupales cognitivoconductuales y conductuales para padres de niños con problemas de conducta, 
concluyen que el entrenamiento de los padres produjo una reducción estadísticamente significativa en los problemas de conducta en los niños, tanto evaluados por los padres como de forma independiente. Y en la misma línea la revisión de Barlow y Stewart-Brown (2000) muestra que el entrenamiento de padres es efectivo en la consecución de cambios positivos y en su mantenimiento en el tiempo.

El formato grupal de intervención con padres ha sido ampliamente utilizado en el tratamiento de niños diagnosticados de trastornos del comportamiento, habiéndose señalado que: es menos costoso, más eficiente, menos estigmatizante que la intervención individual (Chronis, Chacko, Fabiano, Wymbs y Pelham, 2004); favorece las habilidades de organización y reduce el comportamiento oposicionista, disruptivo e inatento en los niños (Korzeniowsk e Ison, 2008; Marinho y Ferreria, 2000); mejora las atribuciones y expectativas respecto a la problemática de los hijos (Roselló, García, Tárraga y Mulas, 2003); incrementa la competencia parental autopercibida (Manjón, 2011); aumenta la frecuencia de emisión de estímulos reforzadores por parte de los padres y aumenta significativamente la frecuencia de interacción y de realización de actividades conjuntas padre-hijo (Marinho y Ferreira, 2000). En la revisión de Zwi, Jones, Thorgaard, York, y Dennis (2011), se analizan cinco estudios con 284 participantes que comparan el entrenamiento para padres de niños diagnosticados de TDAH con el tratamiento habitual de facto, encontrando que el entrenamiento para padres puede tener un efecto positivo sobre el comportamiento de los niños y también sobre la reducción del estrés de los padres, mejorando su confianza.

No obstante, a pesar de los resultados favorables de los estudios sobre la eficacia del entrenamiento de padres y madres, las conclusiones no son inequívocamente positivas (Marinho y Ferreira, 2000). Las investigaciones han demostrado que las desventajas socioeconómicas, los conflictos entre la pareja, los problemas conyugales, los progenitores con síntomas depresivos, el aislamiento de los padres, ser madre soltera y la falta de apoyo social, influyen sobre la participación en el entrenamiento (propensión al abandono prematuro del mismo), sobre la magnitud del cambio terapéutico, sobre el mantenimiento de los cambios a lo largo del tiempo y sobre la satisfacción con el programa de intervención elegido (Furey y Basili, 1988; Kazdin, 1997; McMahon y Forehand, 1983; Webster-Stratton, 1991;), exigiendo que sean adoptadas actitudes eficaces para su superación (Marinho y Ferreira, 2000).

\section{Las terapias contextuales: perspectiva histórica.}

En la Terapia de Conducta se contemplan actualmente tres generaciones de terapias (Hayes, 2004; Pérez Álvarez, 2006; Luciano y Valdivia, 2006) que se diferencian entre sí en cuanto a sus principios conceptuales y terapéuticos. La primera generación, la terapia de conducta clásica, representó un cambio de paradigma en la psicoterapia tradicional, asentando sus bases en la psicología del aprendizaje. Los procedimientos terapéuticos se basan en el manejo de contingen- 
cias para favorecer el cambio conductual, pudiendo entenderse como un modelo contextual e ideográfico en la medida en que atiende a las condiciones antecedentes y consecuentes que se dan en relación con la conducta problema para cada caso particular. Ante las limitaciones de su utilidad en problemas complejos y la imposibilidad de explicar los eventos internos, se erige una segunda generación de psicoterapias, englobadas bajo la denominación de terapias cognitivo-conductuales, que persiguen el cambio en la conducta de las personas sobre la base de un cambio en el contenido de sus eventos cognitivos y emocionales, constituidos como el origen de la psicopatología. Estos modelos se alejan del contextualismo y la perspectiva ideográfica al atribuir los problemas conductuales y emocionales a disfunciones cognitivas específicas de cada tipo de dificultad. Pese a que las intervenciones bajo este marco han sido ampliamente investigadas y han mostrado su eficacia en la generación de cambios, no han dado cuenta del origen, mantenimiento y modificación de las supuestas cogniciones generadoras de sufrimiento en la personas. Es más, la evidencia experimental sugiere que los componentes conductuales, per se, explican la mayor parte de la eficacia de las mismas. La Terapia de Conducta ha alcanzado un tercer desarrollo en el que se rescata la importancia del contexto y de la funcionalidad de la conducta. Se produce un cambio cualitativo en la intervención, dado que se persigue la construcción de repertorios flexibles y efectivos, más que la eliminación de síntomas.

La Terapia de Aceptación y Compromiso (ACT) se enmarca en las terapias contextuales, se asienta en el contextualismo funcional y en la Teoría del Marco Relacional (RFT). La ACT encuadra la experiencia de malestar como parte natural de la vida y orienta la intervención hacia la alteración de la función de los síntomas más que su eliminación, para promover una mayor flexibilidad psicológica y con ello una vida con más significado (Luciano y Valdivia, 2006). El Trastorno de Evitación Experiencial (TEE) (Hayes, Wilson, Gifford, Follete y Strosahl, 1996; Luciano y Hayes, 2001) se refiere a un patrón de comportamiento rígido orientado a la evitación/control del malestar generado por diversos eventos privados aversivos (como recuerdos, pensamientos, sensaciones, emociones) que, pese a que genera un alivio del malestar inmediato, impide construir una vida orientada a los valores personales. Muchos estudios han encontrado que esta dimensión es común a la mayoría de los trastornos psicopatológicos (Ruiz, 2010).

\section{Terapias Contextuales aplicadas al ámbito infantil}

Las investigaciones sobre ACT ofrecen una perspectiva prometedora sobre su eficacia en el abordaje de diversos problemas psicológicos y en su utilidad para minimizar el estigma y eliminar las posibles barreras para comprometerse con un seguimiento psicoterapéutico (Larmar, Wiatrowski y Lewis-Driver, 2014). Existe evidencia sobre la elevada eficacia de protocolos basados en aceptación y valores frente a aquellos que se focalizan en el control del malestar (Ruiz, 2010).

En cuanto a la población infanto-juvenil se están planteando nuevas formas de 
intervención que persiguen objetivos distintos a la terapia de conducta tradicional y que tiene un carácter ideográfico (Ferro, Vives y Ascanio, 2009). Algunos autores como Hayes, Strosahl y Wilson (2014) o Murrell, Coyne y Wilson (2004) han realizado adaptaciones de ACT a este tipo de población. Murrell y Scherbarth (2011) plantean que, al igual que con población adulta, es necesario realizar un análisis funcional del problema y evaluar tanto los valores como los patrones de evitación, empleando como fuentes de información también a los padres, especialmente en niños pequeños. Por otra parte plantean que los componentes de sentido del yo como contexto y de contacto con el momento presente pueden ser muy abstractos para la población infantil y recomiendan el empleo de metáforas y ejercicios adaptados. Existen datos sobre el éxito de intervención de corte contextual para abordar las dificultades emocionales en madres y padres con niñas y niños diagnosticados de autismo (Blackledge y Hayes, 2006); y se muestra como prometedora en la mejora de las relaciones parentales (Coyne y Wilson, 2004). Un ejemplo de ello lo constituye el estudio de Gómez, García, Hódar y Martínez (2012) con familias en riesgo social (negligencia y/o maltrato infantil), que encuentra mejoría en el estilo de funcionamiento parental y en las conductas disfuncionales de los menores tras una intervención en valores basada en ACT. El trabajo de Santamaría y colaboradores (Santamaría, Cebolla, Rodríguez y Miró, 2006) es otro ejemplo de intervención con padres basada en potenciar la autorregulación a través de técnicas de mindfulness con buena eficacia percibida por las madres participantes.

\section{Objetivos y características de la intervención}

En respuesta a la demanda creciente de atención en salud mental en población infantil, queremos describir una propuesta de tratamiento grupal desde el enfoque de ACT, para madres y padres con hijos o hijas que han sido valorados en un Centro de Salud Mental. A la espera de poder evaluar resultados a largo plazo, presentaremos un breve análisis de la valoración realizada por los padres y las madres según la utilidad percibida del grupo.

Los grupos se formaron con progenitores cuyos hijos habían sido evaluados y/o tratados en las consultas de Psicología Clínica de nuestro Centro de Salud derivados tanto desde atención primaria como desde psiquiatría y otras especialidades médicas. Los diagnósticos que presentaban los niños y niñas fueron mayoritariamente trastornos del comportamiento y de las emociones de comienzo habitual en la infancia y adolescencia (trastornos hipercinéticos, disociales, de ansiedad y de vinculación) y de forma más infrecuente, retraso madurativo y síndrome de Asperger. Un criterio de exclusión fue que los menores tuviesen más de 13 años. No se establecieron diagnósticos específicos como criterio de inclusión/ exclusión.

Hemos realizado nueve ediciones de grupos, iniciándose la primera en septiembre de 2012 y la última en septiembre de 2015. El programa se desarrolla 
en 10 sesiones con periodicidad semanal y una hora de duración, estructurándose de acuerdo con el esquema de contenidos descrito en la tabla 1. El número total de participantes, entendiendo como tales los padres y las madres que han acudido al menos a una sesión en cada edición del grupo, y teniendo en cuenta que el padre y la madre del mismo niño o niña se cuentan como una unidad, ascendió a 69 (en adelante usaremos el término "madres" para referirnos a padres y madres, y el término "niños" para referirnos a niñas y niños).

Los teóricos de ACT han resumido en el modelo de inflexibilidad psicológica (Hayes et al., 2014) los diversos procesos que explican el comportamiento problemático y en torno a los cuales gira la terapia. Durante el desarrollo del grupo se ayuda a las madres a que sus acciones sean más congruentes con los valores que quieren que guíen la relación con sus hijos, trabajando los procesos de flexibilidad psicológica. A continuación presentamos en la tabla 1, un resumen de objetivos, contenidos y procesos así como una breve descripción de la adaptación de los diversos componentes terapéuticos de ACT para estos grupos.

Tabla 1.

Cuadro-resumen de los objetivos, contenidos y procesos terapéuticos del grupo

\begin{tabular}{ll}
\hline Sesión & \multicolumn{1}{c}{ Objetivos } \\
\hline 1 & Favorecer un clima \\
terapéutico de colaboración \\
activa. \\
Facilitar explicaciones \\
contextuales del \\
comportamiento de los \\
niños. \\
Clarificar valores comunes \\
en los participantes en \\
relación a la crianza.
\end{tabular}

2 Adquisición de herramientas contextuales para explicar y predecir el comportamiento propio y de los hijos. Contactar emocionalmente con la experiencia de la inutilidad a largo plazo de comportamientos rígidos.

Análisis funcional desde la perspectiva del adulto Análisis funcional desde la perspectiva de los niños
Procesos terapéuticos

Presentación y objetivos del Valores grupo

El papel del contexto en el comportamiento Ejercicio experiencial sobre el rol de madre Metáfora del viaje
Toma de perspectiva
Identificación del patrón de evitación experiencial en las madres Toma de perspectiva Desesperanza creativa Valores
3 Facilitar la observación en vivo (directa o vicariamente) de las consecuencias a corto y largo plazo del comportamiento de las Aceptación Defusión Toma de perspectiva

Representación de
interacciones madre-hijo,
con sus respectivas mentes
Metáfora del autobús

Metáfora del autobús 
madres y de los hijos.

Experimentar la

inevitabilidad de las

emociones negativas en las

interacciones difíciles con

los niños.

4 Facilitar el contacto con la incapacidad de controlar a

Lo que se puede controlar y lo que no

otras personas.

Facilitar la aceptación de eventos privados como emociones negativas, preocupaciones, dudas, etc. como algo humanamente inevitable.

Adquirir habilidad para de-fusionarse.

5 Obtener conocimientos teóricos y habilidades prácticas para aplicar reforzamiento
Ejercicios experienciales acerca del control de eventos privados Metáforas sobre la fusión Ejercicios de distanciamiento

\section{Aceptación}

Defusión
Aceptación

Defusión

Yo contexto
Análisis de las técnicas de refuerzo

Identificación de barreras pasadas y futuras para su aplicación

Ejercicio de defusión
6 Obtener conocimientos teóricos y habilidades prácticas para aplicar la extinción.
7 Obtener conocimientos teóricos y habilidades prácticas para lograr la reducción y eliminación de comportamientos disruptivos.

8 Contactar con las otras áreas valiosas de la vida y tomar conciencia de la influencia que tiene el abandono del cuidado de los propios valores sobre la relación con los hijos.

Elección de compromisos personales para mejorar el sentido de sus propias vidas más allá del rol de madres.
Análisis de la técnica de extinción Identificación de barreras para su aplicación Ejercicios de exposición a la incomodidad

Análisis de las técnicas de castigo

Identificación de barreras para su aplicación Ejercicio experiencial: actuar siendo criticado Ejercicio de defusion

Metáfora del jardín

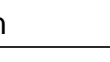

\begin{tabular}{l} 
Aceptación \\
Defusión \\
Acciones \\
orientadas a \\
valores \\
Yo contexto \\
\hline Defusión \\
Toma de \\
perspectiva \\
Acciones \\
orientadas a \\
valores \\
Yo contexto \\
Valores \\
Aceptación \\
Defusión \\
Acciones \\
orientadas a \\
valores
\end{tabular}

Aceptación

orientadas a

valores

Defusión

Toma de

orientadas a

valores

Valores

Aceptación

Defusión

orientadas a 


\begin{tabular}{|c|c|c|c|}
\hline 9 & $\begin{array}{l}\text { Poner en relación la } \\
\text { influencia de las } \\
\text { experiencias previas con los } \\
\text { cuidadores propios con las } \\
\text { dificultades, deseos y } \\
\text { valores en la crianza de los } \\
\text { hijos. } \\
\text { Clarificar valores, distinguir } \\
\text { lo que es importante de lo } \\
\text { que no lo es tanto. }\end{array}$ & $\begin{array}{l}\text { Ejercicio de toma de } \\
\text { perspectiva: identificación } \\
\text { de barreras relacionadas con } \\
\text { la crianza, influencia de las } \\
\text { experiencias con la familia } \\
\text { de origen ("yo como niña vs } \\
\text { yo como madre") } \\
\text { Ejercicio del funeral }\end{array}$ & $\begin{array}{l}\text { Valores } \\
\text { Toma de } \\
\text { perspectiva } \\
\text { Acciones } \\
\text { orientadas a } \\
\text { valores }\end{array}$ \\
\hline 10 & $\begin{array}{l}\text { Facilitar el proceso de } \\
\text { despedida del grupo. } \\
\text { Reflexionar sobre la } \\
\text { experiencia grupal y su } \\
\text { repercusión en el ámbito } \\
\text { familiar y en otros } \\
\text { diferentes. }\end{array}$ & $\begin{array}{l}\text { Ejercicio de mindfulness } \\
\text { Resumen de estrategias, } \\
\text { puesta en común de la } \\
\text { experiencia, cambios } \\
\text { producidos } \\
\text { Despedida y valoración de } \\
\text { la experiencia }\end{array}$ & $\begin{array}{l}\text { Conexión con el } \\
\text { presente } \\
\text { Acciones } \\
\text { orientadas a } \\
\text { valores }\end{array}$ \\
\hline
\end{tabular}

\section{LOS VALORES Y LAS ACCIONES COMPROMETIDAS:}

El trabajo de clarificación de valores con las madres permite que tomen perspectiva de lo que realmente es importante en la relación con sus hijos, así como de su capacidad de transmitir valores. Al comienzo de la terapia, en la primera sesión, como un modo de encuadrar lo que allí se va a hacer, se solicita que pongan en común los valores más importantes que tienen y que querrían transmitir a sus hijos. Éste será el contexto dentro del cual toma sentido el trabajo de aceptación y defusión posterior (Hayes et al., 2014).

Se emplean diversas metáforas como la del viaje (desde la sesión 1) o la del jardín (desde la sesión 8) en diferentes momentos del grupo que permiten remitirnos una y otra vez a los valores para discriminar las interacciones que funcionan y las que no, no según el criterio del terapeuta, sino según el criterio de las propias madres. Esto permite salir del enredo que supone establecer una dinámica de preguntas-respuestas al terapeuta acerca de qué hacer en cada momento como si hubiese una solución inmediata y fácil que resolviera cada conflicto.

La confusión entre valores y objetivos potencia la rigidez en los patrones de comportamiento de las madres. El problema de confundir objetivos ("quiero que se vista solo") con valores ("quiero que sea autónomo") supone una elevada frustración cuando una y otra vez no se consigue el objetivo pese a haber depositado muchos esfuerzos en lograrlo (pedirle muchas veces que se vista, negociar, regañar...). Pero además no es infrecuente que la importancia de ese objetivo sea mucho menor en comparación con las consecuencias dañinas que genera el seguir intentando conseguirlo de forma rígida, "a cualquier precio" (repitiendo todos los días un intercambio de críticas, regañinas, amenazas...). Mediante el análisis funcional que se trabaja principalmente en las sesiones 2 y 3 , las madres caen en la 
cuenta de su patrón rígido orientado a "resolver a la corta" y sentirse mejor (vistiéndolo para salir a tiempo de casa), perdiendo de vista que a la larga se está invirtiendo en fomentar lo que no se quiere (que continúe siendo dependiente).

La metáfora del viaje nos permite incluir elementos que remarcan las dificultades o los obstáculos que pueden aparecer en un viaje y comenzar a introducir claves que faciliten tanto la aceptación como el distanciamiento. Por ejemplo, les planteamos que a veces en el camino hacia el norte (en equivalencia con los valores que han puesto en común: "respetar a los demás", "ser trabajadora", "valerse por una misma", "ser bueno con los demás"...) llueve, hay caminos cortados, carreteras sin asfaltar, etc. y eso nos tienta a cambiar el rumbo e ir por otro camino más fácil, pero que nos lleva a un sitio que no es el que queremos (nos lleva al sur, a lo opuesto a los valores señalados). Las acciones valiosas son aquéllas que permiten avanzar en dirección norte, pese a los obstáculos. Durante un viaje largo como es el de la crianza de los hijos es muy difícil no desviarse nunca del rumbo. Esta metáfora nos permite no quedarnos estancados en el error sin posibilidad de cambio. Cuando caen en la cuenta de algún error y nos piden respuesta, les preguntamos, "¿qué hacéis si yendo camino a la playa os dais cuenta de que os habéis desviado?","¿volvéis a casa y os quedáis sin vacaciones?", etc. De esta forma, a través de las relaciones de equivalencia entre los elementos de la metáfora y el problema, son las propias madres quienes encuentran respuestas.

Enmarcado dentro de las acciones comprometidas con los valores repasamos con las madres en sesiones más avanzadas $(5,6$ y 7$)$ las cuestiones teóricas sobre modificación de conducta (como las técnicas de refuerzo, extinción, control de contingencias como los sistemas de puntos, etc.). En este momento nos encontramos con que la mayoría han probado a aplicar alguna de estas técnicas básicas y sin embargo es excepcional que lo hayan hecho adecuadamente, siguiendo las leyes del aprendizaje. Es habitual que hayan aplicado sistemas de puntos de formas desproporcionadas e inconsistentes; o que en un intento de aplicar extinción terminen invalidando las emociones del niño y reforzando comportamientos disruptivos mucho más intensos.

Suele haber dos momentos muy significativos en la terapia. El primero es el trabajo con la metáfora del jardín en la sesión 8, que amplía la perspectiva para que sean conscientes de cómo está su vida, en sus diferentes ámbitos significativos (según sus valores, cómo están las "plantas" de la salud, de la pareja, de la amistad, del trabajo, del ocio, etc...); y la relación que todo ello tiene en cómo se comportan con sus hijos, en las expectativas y deseos que depositan en ellos. A través de esa metáfora aparecen más obstáculos o "malas hierbas" (como "no tengo tiempo para eso", "ya lo haré cuando crezcan los niños", "ahora me tengo que centrar en el problema del niño", "no me apetece") y la posibilidad de volver o empezar a "regar" las plantas importantes. El segundo de ellos es el ejercicio del funeral en la sesión 9 , en el que se enfrentan de un modo emocionalmente intenso a la discriminación de lo que realmente es importante y lo que no lo es tanto, de lo que verdaderamente 
quieren hacer con sus vidas y lo que están haciendo.

\section{EL CONTROL ES EL PROBLEMA. ACEPTACIÓN}

A través del análisis funcional que realizamos desde la sesión 2 se facilita la discriminación del efecto a corto y a largo plazo de lo que hacen las madres. En la mayoría de conflictos entre madres e hijos podemos identificar con facilidad un patrón de evitación experiencial tanto en el niño como en las madres. La diferencia es que un niño no tiene adquirida la capacidad de ver la repercusión de sus actos en un futuro lejano, las madres sí y por ello utilizamos ese recurso motivacional, poniendo en relación de oposición lo que hacen ahora con el resultado que querrían tener no sólo ahora sino a medio y largo plazo.

La segunda sesión la dedicamos al análisis funcional de las interacciones hijos-madres. Identificar los eventos privados que pueden estar al mando de la conducta de las madres es fundamental para que sean conscientes de que en muchas ocasiones su comportamiento no se orienta a sus valores sino que funciona como una forma de escapar de esos sentimientos/pensamientos/sensaciones, de aliviar su tensión interna. Se remarca entonces el efecto que tendrá esto a largo plazo en la relación con los niños, anticipando si las situaciones mejoran o empeoran. Por ejemplo: se les pregunta si sería congruente pedir a un hijo que haga algo (por ejemplo: "hablar sin gritar aunque esté enfadada") al tiempo que ellas mismas no lo hacen. Este tipo de preguntas facilita que caigan en un estado emocional que se ha denominado desesperanza creativa, ya que es un motor emocional para un cambio creativo, nuevo, diferente.

Bien pronto en el desarrollo del grupo tenemos que lograr el objetivo de que las madres puedan convivir con el malestar que les genera el darse cuenta de que al ser los niños seres humanos diferentes de ellas, no hay nada que puedan hacer que asegure que tendrán un control absoluto sobre ellos. Las madres vienen con sus ideas sobre el mundo, reglas acerca de cómo deberían ser las cosas, experiencias propias con sus familias de origen, etc. El hacer el análisis funcional del comportamiento del niño desde su perspectiva, teniendo que hipotetizar cómo piensan o sienten antes y después de lo que hacen las madres, facilita que entiendan mejor las reacciones de sus hijos. Ejercicios como el rol playing de la sesión 3 en el que se representa al niño y a su mente y a la madre y a su mente, ayudan a poner de relieve de forma más experiencial las vivencias internas de los niños.

Aprovechando la exposición sobre las técnicas de modificación de conducta que realizamos en las sesiones 5,6 y 7 , se realizan ejercicios experienciales que activan los obstáculos psicológicos que se presentarán en casa a la hora de llevarlas a la práctica (pensamientos como "no debería estar pasando esto", "no puedo más", "no va a funcionar con mi hijo", "soy mala madre"; emociones como "rabia", "desesperación", "miedo"; sensaciones como "tensión", "cansancio"; recuerdos como "yo nunca contesté así a mi madre", etc.). De este modo favorecemos la aceptación de ese malestar, estableciendo que la aparición de estos eventos privados 
puede ser la señal de que están aplicando adecuadamente la técnica ("a veces ir hacia el norte hace que te sientas mal en ese tramo, pero al final del día has avanzado unos pocos kilómetros").

Hacia el final del grupo, en la sesión 9, les proponemos un ejercicio en el que recuerdan cómo eran ellas con la edad de sus hijos, cómo las cuidaron a ellas, lo que les ayudó y lo que no de lo que les enseñaron sus padres, etc. Es inevitable que la propia historia con la familia de origen genere reglas acerca de cómo hacer o no hacer con los hijos. Esta perspectiva ayuda también a notar que son diferentes de sus hijos, que los niños tienen sus propios deseos y necesidades que no son las suyas. Cuando las madres se dan cuenta de estas influencias y son capaces de aceptar el malestar relacionado con la crianza, se vuelven más flexibles y capaces de elegir cómo relacionarse con sus hijos.

\section{EL MOMENTO PRESENTE, LA DEFUSION Y EL YO COMO CONTEXTO}

El trabajo con las propias barreras de las madres junto con la clarificación de valores es el núcleo de la terapia. La clarificación de valores puede encajar mejor sus narrativas acerca de lo que se espera del grupo, sin embargo el comenzar hablando de sus emociones o pensamientos les resulta chocante. Además de las metáforas del viaje y el jardín, la metáfora del autobús que trabajamos desde la sesión 3 es muy útil en la identificación de barreras (pasajeros molestos como pensamientos o emociones aversivas) así como para generar distanciamiento de las mismas y potenciar la función de mando del yo ("el conductor es el único que puede finalmente decidir por dónde quiere conducir, puede elegir la dirección pese a que los pasajeros no quieran que siga ese camino").

Durante el desarrollo de las sesiones, desde la cuarta, se van introduciendo ejercicios de mindfulness como un modo de conectarse con el presente, sin dejarse arrastrar por los "pasajeros del autobús". Se introducen ejercicios para diferenciar de forma experiencial en qué momentos estamos fusionados y en qué otros defusionados y conectados con el presente, como con el ejercicio de mirar el dedo índice e ir cambiando el foco atencional.

El yo contexto se trabaja a través de metáforas en las que se potencia la jerarquía del yo con respecto a los eventos privados como la metáfora del autobús, la del cielo y las nubes, la del ajedrez, etc. Al inicio del grupo se realizan ejercicios más sencillos, según se progresa se expone a las madres a estímulos que evocan reacciones emocionales más aversivas. Por ejemplo, en la sesión 6 , se realiza el ejercicio de la postura incómoda en el cual, habiendo generado un contexto de valor, se les pide que prueben a permanecer en esa postura abiertas a la experiencia. Las participantes establecen después equivalencias entre esas sensaciones de dolor y otras experiencias aversivas de su vida ("me siento con rabia cuando el niño me reta") y su capacidad para elegir qué hacer. Hacer el ejercicio en la misma sesión en la que se analiza la técnica de la extinción facilita que se vean a sí mismas capaces de mantenerse en un comportamiento (por ejemplo, no gritar al niño ante un 
comportamiento disruptivo) pese a que estén sintiendo malestar (por ejemplo, mucha tensión, enfado por lo que hace el niño...) que empuja a liberar la tensión o el malestar (por ejemplo, gritándole, pidiendo que pare, ofreciéndole lo que pide...).

\section{Resultados}

En relación a la adherencia al tratamiento, se puede observar la distribución de la participación por grupos en la tabla 2. El porcentaje de participantes que abandonó el grupo fue del 14.5\%, es decir, 10 progenitores de los 69 iniciales, entendiendo como abandono el no haber acudido al menos a la mitad de las sesiones. Por tanto, el porcentaje de retención es del $85.5 \%$, es decir, 61 padres y madres mantienen su asistencia al grupo. Además, de los 69 participantes iniciales, el $73.9 \%$ acuden al menos al $70 \%$ de las sesiones.

\section{Tabla 2.}

Grado de adherencia de los participantes.

\begin{tabular}{|c|c|c|c|c|c|}
\hline Grupo & $\begin{array}{l}\text { Participantes } \\
\text { totales (en } \\
\text { alguna } \\
\text { sesión) }\end{array}$ & $\begin{array}{l}\text { Participantes } \\
\text { que acudieron } \\
\text { al menos al } \\
50 \% \text { de las } \\
\text { sesiones }\end{array}$ & $\begin{array}{l}\text { Porcentaje de } \\
\text { participantes } \\
\text { que acudieron } \\
\text { al } 50 \% \text { de las } \\
\text { sesiones }\end{array}$ & $\begin{array}{l}\text { Participantes } \\
\text { que acudieron } \\
\text { al } 70 \% \text { de las } \\
\text { sesiones }\end{array}$ & $\begin{array}{l}\text { Porcentaje de } \\
\text { participantes } \\
\text { que acudieron } \\
\text { al } 70 \% \text { de las } \\
\text { sesiones }\end{array}$ \\
\hline A & 10 & 8 & $80 \%$ & 8 & $80 \%$ \\
\hline$B$ & 8 & 7 & $87,5 \%$ & 5 & $62,5 \%$ \\
\hline $\mathrm{C}$ & 7 & 6 & $85,7 \%$ & 6 & $85,7 \%$ \\
\hline $\mathrm{D}$ & 6 & 6 & $100 \%$ & 6 & $100 \%$ \\
\hline$E$ & 8 & 5 & $62,5 \%$ & 5 & $62,5 \%$ \\
\hline $\mathrm{F}$ & 5 & 5 & $100 \%$ & 4 & $80 \%$ \\
\hline $\mathrm{G}$ & 6 & 6 & $100 \%$ & 4 & $66,7 \%$ \\
\hline $\mathrm{H}$ & 11 & 9 & $81,8 \%$ & 6 & $54,5 \%$ \\
\hline 1 & 8 & 8 & $100 \%$ & 7 & $87,5 \%$ \\
\hline
\end{tabular}

Por otra parte, respecto a la valoración de la utilidad del grupo, un total de 52 progenitores la completaron de forma anónima al finalizar el grupo. En el gráfico 1 se muestra la valoración media de cada grupo. Los participantes valoraron de media con un 8.9 sobre 10 la utilidad del grupo en el ámbito de la relación y crianza de sus hijos. Asimismo, valoraron de media con 8.5 sobre 10 la utilidad del grupo para otras áreas de su vida que no tenían que ver con la crianza ni con el problema que motivaba inicialmente la consulta. 


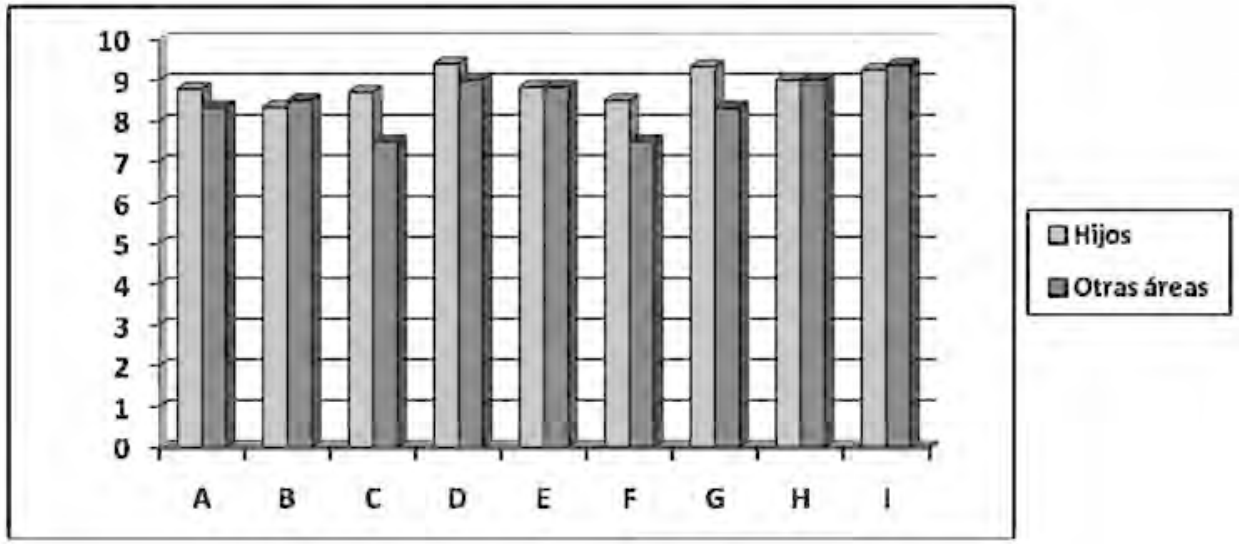

Gráfico 1.

Utilidad percibida de la intervención en el ámbito de los hijos y en otros ámbitos de la vida

\section{Discusión y conclusiones}

La impresión de que la normalidad del comportamiento de los niños se va restringiendo progresivamente, nos llevó a diseñar una intervención que tuviera en cuenta la importancia del contexto en el que se producen tales comportamientos. La intervención orientada por diagnósticos tal vez contribuya a potenciar la atribución interna de problemas mentales en niños y a la conceptualización medicalizada de su comportamiento, por ejemplo, "tiene TDAH", como si en sí mismo el diagnóstico explicase lo que le ocurre (García de Vinuesa, Pérez Álvarez y González Pardo, 2014). Las madres en ocasiones se han sentido culpadas por distintas personas por el comportamiento de sus hijos, lo cual favorece que acojan los diferentes diagnósticos realizados a sus niños con un alivio inmenso, pero esto les puede desconectar de su papel nuclear en el desarrollo del niño. Con la orientación de la ACT pretendemos ayudar a que las madres tomen conciencia del efecto de lo que ellos hacen en relación a sus hijos, a la corta y a la larga, rescatando el abordaje idiográfico y funcional en la explicación y abordaje de los problemas.

Frente a los enfoques psicoeducativos basados en la enseñanza de técnicas conductuales, decidimos orientar el grupo desde ACT dado que en la práctica clínica hemos observado, al igual que otros autores (Coyne y Murrell, 2009), que la falta de entendimiento de las leyes de aprendizaje que subyacen a las técnicas conductuales, hace que sean empleadas de forma errónea manteniendo o generando problemas. Por ello para nosotros tenía más sentido enmarcar estas técnicas en un tratamiento orientado a clarificar lo que es realmente importante y lo que no, según los valores de cada familia, y a adquirir habilidades para relacionarse de forma diferente con su malestar, de modo que les permita mejorar la relación con los hijos (y así la forma en que los hijos se relacionan con el suyo propio). Bajo esta perspectiva el terapeuta no da pautas, fomenta un aprendizaje experiencial que 
potencie el papel protagonista de los progenitores, son ellas y ellos quienes eligen qué hacer.

Uno de los efectos que consideramos fundamentales del grupo es la adquisición de un mejor entendimiento del sentido que pueden tener los comportamientos de los niños en los diferentes contextos. Aprenden a analizarlo funcionalmente, se dan cuenta de que los hijos tienen su propia perspectiva, sus propios eventos privados, etc. Desde otras perspectivas se podría decir que los participantes aprenden a "mentalizar" mejor en relación a los hijos (Bateman y Fonagy, 2012). Por todo ello, este abordaje podría ser un acercamiento puente entre las orientaciones más "democráticas" o "basadas en las relaciones interpersonales", que destacan la comunicación y los procesos de interacción entre los miembros de la familia y las "conductuales" (Barlow, Coren y Stewart-Brown, 2002; Richardson y Joughin, 2002).

Los resultados que hemos mostrado apuntan que el enfoque grupal orientado desde ACT permite trabajar procesos de cambio pese a las especificidades de cada caso, con madres de niños de diferentes edades, con diferentes diagnósticos y con contextos familiares y socioeconómicos distintos. Como se señaló anteriormente, las investigaciones han demostrado que factores relacionados con el sufrimiento de los progenitores (como los conflictos entre la pareja, los problemas conyugales, el tener síntomas depresivos, el aislamiento, ser madre soltera, los problemas socioeconómicos, la falta de apoyo social) afectan a la participación en las terapias (propensión al abandono prematuro del mismo), a la magnitud y duración del cambio terapéutico y a la satisfacción con el programa de intervención elegido (Furey y Basili, 1988; Kazdin, 1997; McMahon y Forehand, 1983; WebsterStratton, 1991). Por ello, dado que la utilidad percibida es elevada no sólo en relación a los problemas con los hijos sino, prácticamente al mismo nivel, en otros ámbitos de su vida, creemos que esta orientación puede mejorar la eficacia de la intervención en casos donde enfoques clásicos no han podido dar respuesta, ofreciendo una intervención con un buen equilibrio costes/beneficios.

Además hemos conseguido tasas de participación altas. La tasa de abandono en nuestros grupos, habiendo definido un criterio restrictivo como lo es el no haber acudido al menos a la mitad de las sesiones, es inferior a la encontrada en algunos grupos de intervención con padres como el de Marinho y Ferreira (2000), que ascendió a 22,7\%, o a otros grupos relatados en la literatura, que oscilan entre el $30 \%$ y el 60\% (Kazdin, 1994, citado por Marinho y Ferreira, 2000).

Finalmente, comentarios como "me acordé mucho de vosotros esta semana porque tenía a los pasajeros del autobús muy alborotados, respiré y logré no desviarme al sur", "ahora me doy cuenta de lo que es importante y de lo que no lo es tanto", "al verle reaccionar así pensé que estaría muy disgustado, que algo le habría pasado en el colegio", nos muestran cómo los participantes, según transcurren las sesiones, son más capaces de relacionarse de forma diferente con sus emociones, recuerdos, sensaciones y pensamientos, y con esa distancia pueden 
entender mejor a sus hijos y elegir qué quieren hacer con ellos (y con sus vidas). Esta experiencia como terapeutas sin duda nos anima a continuar el viaje pese a los obstáculos.

\section{Referencias bibliográficas}

Barlow, J., Coren, E., \& Stewart-Brown, S. (2002). Meta-analysis of the effectiveness of parenting programmes in improving maternal psychosocial health. The British Journal of General Practice, 52(476), 223-233.

Barlow, J., \& Stewart-Brown, S. (2000). Behavior problems and group-based parent education programs. Journal of developmental \& behavioral pediatrics, 21(5), 356-370.

Bateman, A., Fonagy, P. (2012) (eds.) Handbook of Mentalizing in Mental Health Practice. Washington: American Psychiatric Publishing, Inc.

Blackledge, J. T., \& Hayes, S. C. (2006). Using Acceptance and Commitment Therapy in the support of parents of children diagnosed with autism. Child \& Family Behavior Therapy, 28(1), 1-18.

Brestan, E. V., \& Eyberg, S. M. (1998). Effective psychosocial treatments of conduct-disordered children and adolescents: 29 years, 82 studies, and 5,272 kids. Journal of clinical child psychology, 27(2), 180-189.

Chronis, A. M., Chacko, A., Fabiano, G. A., Wymbs, B. T., \& Pelham, W. E. (2004). Enhancements to the standard behavioral parent training paradigm for families of children with ADHD: Review and future directions. Clinical Child and Family Psychology Review, 7, 1-27.

Coyne, L.W.\& Murrell, A.R. (2009). The joy of parenting. An acceptance \& commitment therapy guide to effective parenting in the early years. Oakland: New Harbinger Publications.

Coyne, L.W. \& Wilson, K. G. (2004). The role of cognitive fusion in impaired parenting: An RFT analysis. International Journal of Psychology and Psychological Therapy, 4, 469-487.

Ferro, R., Vives, C., \& Ascanio, L. (2009). Novedades en el tratamiento conductual de niños y adolescentes. Clínica y Salud, 20(2), 119-130.

Forehand, R. L., \& McMahon, R. J. (1981). Helping the noncompliant child: A clinician's guide to parent training. New York: Guilford Press.

Furey, W. M., \& Basili, L. A. (1988). Predicting consumer satisfaction in parent training for noncompliant children. Behavior Therapy, 19(4), 555-564.

Furlong, M., McGilloway, S., Bywater, T., Hutchings, J., Smith, S. M., \& Donnelly, M. (2012). Behavioral and cognitive-behavioural group-based parenting interventions for early-onset conduct problems in children age 3-12 years. Cochrane Database of Systematic Reviews, 2.

García de Vinuesa, F., Pérez Álvarez, M., \& González Pardo, H. (2014). Volviendo a la normalidad. La invención del TDAH y del trastorno bipolar infantil. Madrid: Alianza Editorial.

Gómez, I., García, M.R., Hódar, J.C., \& Martínez, M.M. (2012). Intervención en valores con familias de riesgo social desde la Terapia de Aceptación y Compromiso. Análisis y Modificación de Conducta, 38(157-158), 39-58.

Hayes, S.C. (2004). Acceptance and commitment therapy, relational frame theory, and the third wave of behavioral and cognitive therapies. BehaviorTherapy, 35, 639-665.

Hayes, S.C., Strosahl, K., \& Wilson, K. (2014). Terapia de Aceptación y Compromiso. Proceso y práctica del cambio consciente (Mindfulness). Bilbao: Desclée de Brouer.

Hayes, S.C., Wilson, K.G., Gifford, E.V., Follette, V.M., y Strosahl, K. (1996). Experiential avoidance and behavior disorder: A functional dimensional approach to diagnosis and treatment. Journal of Consulting and Clinical Psychology, 64, 1152-1168.

Kazdin, A. E. (1997). Parent management training: Evidence, outcomes, and issues. Journal of the American Academy of Child \& Adolescent Psychiatry, 36(10), 1349-1356.

Korzeniowks, C., \& Ison, M. (2008). Estrategias psicoeducativas para padres y docentes de niños con TDAH. Revista Argentina de Clínica Psicológica, 18(1), 65-71.

Larmar, S., Wiatrowski, S., \& Lewis-Driver, S. (2014). Acceptance \& Commitment Therapy: An Overview of Techniques and Applications. Journal of Service Science and Management, 7,216-221.

Luciano, C., \& Hayes, S. C. (2001). Trastorno de evitación experiencial. Revista Internacional de Psicología Clínica y de la Salud, 1, 109-157. 
Luciano, C., \& Valdivia, M.S. (2006). La Terapia de Aceptación y Compromiso (ACT). Fundamentos, características, evidencia. Papeles del Psicólogo, 27(2), 79-91.

Manjón, I. (2011). Grupo psicoeducativo a padres de niños con problemas de conducta. ISEP Science, 1, 24-36.

Marinho, M. L., \& Ferreira, E. (2000). Evaluación de la eficacia de un programa de entrenamiento de padres en grupo. Psicología Conductual, 8(2), 299-318

McMahon, R. J., \& Forehand, R. L. (1983). Consumer satisfaction in behavioral treatment of children: Types, issues, and recommendations. Behavior Therapy, 14(2), 209-225.

Murrell, A. R., Coyne, L. W., \& Wilson, K. G. (2004). ACT with children, adolescents, and their parents. In A practical guide to acceptance and commitment therapy (pp. 249-273). US: Springer.

Murrell, A,R., \& Scherbarth, A.J. (2011). State of the research \& literature address: ACT with children, adolescents and parents. The International Journal of Behavioral Consultation and Therapy, 7(1),15-22.

Olivares, J., Macià, D., \& Méndez, F. X. (1993). Intervención comportamental-educativa en el entrenamiento a padres. En D. Macià, F. Méndez, \& J. Olivares (Eds.), Intervención psicológica: Programas aplicados de tratamiento (pp. 115-146). Madrid: Pirámide.

Patterson, G. R. (1974). Interventions for boys with conduct problems: multiple settings, treatments, and criteria. Journal of Consulting and Clinical Psychology, 42(4), 471-481.

Pérez Álvarez, M. (2006). La terapia de conducta de tercera generación. eduPsykhé 5(2), 159-172.

Plaza, I. M. (2011). Grupo psicoeducativo a padres de niños con problemas de conducta. ISEP Science, 1, 24-36.

Robles, Z. y Romero, E. (2011) Programas de entrenamiento para padres de niños con problemas de conducta: una revisión de su eficacia. Anales de Psicología, 27( 1), 86-101.

Rey, C.A. (2006). Entrenamiento de padres: una revisión de sus principales componentes y aplicaciones. Revista infancia, adolescencia y familia, 1(1), 61-84

Richardson, J., \& Jougghin, C. (2002). Parent training programmes for the management of young children with conduct disorders. Findings from research. London: Gaskell.

Roselló, B., García Castellar, R., Tárraga Mínguez, R., \& Mulas, F. (2003). El papel de los padres en el desarrollo y aprendizaje de los niños con trastorno por déficit de atención con hiperactividad. Revista de Neurologia, 36(Supl. 1), 79-84.

Ruiz, F.J. (2010). A review of Acceptance and Commitment Therapy (ACT) empirical evidence: correlational, experimental psychopathology, component and outcome studies. International Journal of Psychological Theraphy, 10(1), 125-162.

Sanders, M. R., \& Dadds, M. R. (1993). Behavioral family intervention. Needham Heights, MA, US: Allyn \& Bacon.

Santamaría, M.T., Cebolla, A., Rodríguez, P. J., \& Miró, M. T. (2006). La práctica de la meditación y la atención plena: técnicas milenarias para padres del siglo XXI. Revista de Psicoterapia, 17(66-67), 157-175. http:// revistadepsicoterapia.com/la-practica-de-la-meditacion-y-la-atencion-plena-tecnicas-milenarias-para-padres-del-siglo-xxi.html

Silvares, E. F. D. M. (1995). O modelo triádico no contexto de terapia comportamental com familias. Psicologia: teoria e pesquisa, 11(3), 235-241.

Webster-Stratton, C. (1991). Annotation: Strategies for helping families with conduct disordered children. Journal of Child Psychology and Psychiatry, 32(7), 1047-1062.

Webster-Stratton, C. (1994). Advancing videotape parent training: A comparison study. Journal of Consulting and Clinical Psychology, 62(3), 583-593.

Webster-Stratton, C. (1996). Early-onset conduct problems: does gender make a difference? Journal of Consulting and Clinical Psychology, 64(3), 540-551.

Webster-Stratton, C. y Hammond, M. A. (1997). Treating children with early onset conduct problems: A comparison of child and parent training interventions. Journal of Consulting and Clinical Psychology, 65(1), 93-109

Zwi, M., Jones, H., Thorgaard, C., York, A., \& Dennis, J. A. (2011). Parent training interventions for Attention Deficit Hyperactivity Disorder (ADHD) in children aged 5 to 18 years. The Cochrane Library. 